\title{
La calidad del servicio en la mejora de la gestión en la atención al usuario de CNEL EP del cantón Milagro- Ecuador en el año 2018.
}

The quality of the service in the improvement of the management in the attention to the user of CNEL EP of the canton Milagro-Ecuador in the year 2018.

Luis Hernán Campos Freire ${ }^{1} \&$ Carlos María Lazo Vento. ${ }^{2}$

DOI: https://doi.org/10.33262/visionariodigital.v3i2.395

\section{Resumen.}

La globalización, tecnología, capacidad y nuevos roles del talento humano en las empresas públicas amerita que se debe cumplir con servicios de calidad, para lo cual debe contar con herramientas necesarias que le permita alcanzar niveles óptimos de satisfacción de los usuarios. Una de estas herramientas es la gestión de calidad en sus procesos administrativos, en el que se enmarque en adiestrar al talento humano identificado con la filosofía de calidad.

Implementar un modelo para mejorar la calidad de servicio a los usuarios de CNEL EP es la clave para que la institución eleve y mantenga su prestigio ante la comunidad a la que se debe, la única forma de realizar es ofrecer compromiso de calidad, asegurando un futuro efectivo y de confianza mediante un modelo administrativo bien planeado y documentado.

La investigación centra su importancia en establecer el proceso por el cual la institución puede pasar a un estado de mejora no solo de calidad sino en mejora continua de atención y servicio al usuario. Es por ello que, cuando se pretende mejorar la calidad de los servicios que CNEL EP entrega a sus usuarios, fortalecer las capacidades de gestión de la institución, instalar una cultura de mejoramiento de los procesos, basada en la autoevaluación y la aplicación de planes o iniciativas de mejora como camino para llegar a la excelencia, este tipo de modelo aparece como herramienta válida para entregar una referencia de la gestión de los servicios.

Palabras claves: Calidad, gestión, mejora, satisfacción del usuario.

\footnotetext{
${ }^{1}$ Universidad Estatal de Milagro, Milagro, Ecuador, hcamposf2011@hotmail.com

${ }^{2}$ Universidad Estatal de Milagro, Milagro, Ecuador, clazov@unemi.edu.ec
} 


\begin{abstract}
.
The globalization, technology, capacity and new roles of human talent in public companies warrants that quality services must be met, for which it must have the necessary tools to achieve optimal levels of user satisfaction. One of these tools is the management of quality in its administrative processes, which is framed in training human talent identified with the philosophy of quality.

Implementing a model to improve the quality of service to users of CNEL EP is the key for the institution to elevate and maintain its prestige in the community to which it is owed, the only way to do it is to offer quality commitment, ensuring a future effective and trustworthy through a well-planned and documented administrative model.

The research focuses its importance on establishing the process by which the institution can move to a state of improvement not only in quality but in continuous improvement of attention and service to the user. That is why, when it is intended to improve the quality of the services that CNEL EP delivers to its users, strengthen the management capabilities of the institution, install a culture of process improvement, based on self-assessment and the application of plans or improvement initiatives as a way to reach excellence, this type of model appears as a valid tool to provide a reference for the management of services.
\end{abstract}

Keywords: Quality, management, improvement, user satisfaction.

\title{
Introducción.
}

El presente artículo hace referencia a un modelo para mejorar la calidad de atención como instrumento de apoyo a CNEL EP que quiere alcanzar resultados de excelencia, es factible y, el mismo servirá como marco de referencia que ayude a las demás instituciones públicas a planificar y evaluar su desempeño o rendimiento en una amplia variedad de procesos e indicadores, orientar y alinear los recursos e iniciativas institucionales para avanzar en el mejoramiento de la gestión y logro de las metas estratégicas.

El modelo propuesto es flexible y no prescriptible, es decir, permite a cualquier tipo de organización utilizar y adaptar según sus necesidades. La propuesta se basa en el principio de gestión orientada hacia la satisfacción de los usuarios, la calidad es intrínseca al servicio y es el usuario de CNEL EP quien la juzga a partir de percepciones por lo tanto la institución debe estar atenta a todas las características y atributos del servicio brindado, son estos los que aumenta o no su satisfacción, determinan sus preferencias y los hace creer en la excelencia del servicio público.

Es viable la investigación, basados en la teoría del capital intelectual, corresponden a elementos constitutivos del proceso de atención en CNEL EP. Estos hacen referencia a la calidad en términos de la atención por parte del funcionario (humano); calidad en términos de agilidad en los procesos y tiempo de espera (organizativo de los procesos); y calidad en términos de infraestructura que permite un buen servicio (espacio físico) todos explicarían en mayor o menor medida la calidad del servicio entregado (calidad percibida) y a la imagen que proyecta CNEL EP (imagen), en conjunto determinan el grado de 
bienestar del usuario en relación a la experiencia al acudir a la institución, es el constructor de satisfacción.

\section{Desarrollo.}

El método de investigación que se desarrolló en esta investigación es de tipo documental y de observación haciendo mucho énfasis en la realización de un análisis crítico sobre la problemática a tratar, recogida en diferentes fuentes como: materiales complementarios, textos, tesis de maestría, doctorados, resoluciones y publicaciones.

Según Huergo (2016) la palabra gestión proviene de "gestus", una palabra latina que significa: actitud, gesto, movimiento del cuerpo. En principio, este significado remite a lo que el sociólogo Pierre Bourdieu ha designado la hexis, esto es: el modo en que un hábitus (una serie de esquemas, dispositivos e interpelaciones culturales internalizadas por los sujetos) se expresa a través del cuerpo en gestos, posiciones, movimientos, etc. Pero este significado no dice nada sobre el carácter activo de la gestión, ya que pone énfasis en movimientos y actitudes vividas como "naturales" por los sujetos de una determinada cultura. Sin embargo, "gestus" es derivada de otra palabra latina: "gerere", que posee varios significados:

- Llevar adelante o llevar a cabo,

- Cargar una cosa,

- Librar una guerra o trabar combate,

- Conducir una acción o un grupo,

- Ejecutar, en el sentido de un artista que hace algo sobre un escenario.

La palabra gestión proviene directamente de "gestio-onis": acción de llevar a cabo y, además, está relacionada con "gesta", en tanto historia de lo realizado, y con "gestación", llevar encima. La gestión implica también una concepción y una práctica respecto del poder, de la administración y la circulación del mismo y de las formas de construir consensos y hegemonías dentro de una determinada organización o institución. Vale recalcar que la construcción de hegemonías no significa inmediatamente el planteamiento de situaciones de dominio, sino la posibilidad y el proyecto de articulación de fuerzas y de diferencias, a través de un imaginario y un objetivo común (Uranga, 2015).

Según Aguilar, (2014) la Gestión (o Management) es la actividad que desarrollan los directivos en el seno de una empresa u organización. Son los encargados de conseguir un nivel adecuado de eficiencia y productividad. Aunque resulte paradójico, los directivos no desarrollan trabajo en el sentido ordinario de la palabra; lo que hacen es realizar para la organización cinco funciones y, al hacerlo, representan tres importantes papeles y aplican otras tantas capacidades primordiales.

Aguilar considera que "Su grado de efectividad no viene dado por sus esfuerzos personales sino por los resultados que alcancen. No tienen que ceñirse a planteamientos teóricos, sino que tienen que tener la habilidad de saber escoger y aplicar los métodos o 
técnicas que sean más apropiadas a una situación real determinada" (Aguilar, 2014, pág. $5)$.

Por todo ello, (Campuzano, Ziadet, \& Echeverría, 2016) definen los cinco conceptos claves relacionados con la Gestión son:

1. Los directivos desarrollan unas tareas y servicios diferentes con respecto al resto de las personas que integran la organización.

2. Al llevar a cabo su trabajo, los directivos realizan para la empresa cinco funciones características: Planificar, Organizar, Dotar de personal, Dirigir y Controlar.

3. Los directivos representan tres importantes papeles en la organización (Interpersonal, Informativo y Decisorio) y aplican tres aptitudes básicas (Intelectuales, de Relaciones humanas y Técnicas).

4. La efectividad de los directivos se juzga en función de los resultados que obtengan para la organización en función de los recursos disponibles.

5. Los directivos tienen que ser capaces de discernir las diferencias que existen entre distintas situaciones y aplicar aquellos métodos que sean más convenientes según los factores que predominen en cada situación específica.

"La Gestión (o Management) es el proceso en virtud del cual se manejan una variedad de recursos esenciales con el fin de alcanzar los objetivos de la organización" (Majad, 2016, pág. 150). Uno de los recursos más importantes son las personas que trabajan para la empresa. Los directivos dedican gran parte de sus esfuerzos a planificar, dirigir y controlar el trabajo de estos recursos humanos. La diferencia entre los directivos y el resto de los empleados está en que los primeros dirigen el trabajo de los demás (Peláez, 2014).

La efectividad de la Gestión consiste en mantener un adecuado equilibrio entre los dos factores: resultados y recursos. Lo ideal es obtener los máximos resultados con los mínimos recursos, pero esto no siempre es posible, por lo que es más realista buscar la eficiencia persiguiendo unos resultados óptimos, no máximos. En este caso, óptimo significa el resultado más favorable bajo unas circunstancias específicas o limitadas, es decir, los resultados dependerán de la adecuación, eficacia y utilización de los recursos empleados (Aduna \& García, 2014).

Hay que tener en cuenta además que existen pocas situaciones exactamente iguales. En todas suelen intervenir un alto número de factores diferenciadores, tales como el grado de motivación y capacidad de los participantes, los recursos financieros y materiales disponibles, la claridad de las comunicaciones, la cantidad de datos o información accesible, y los grados de importancia y urgencia de la propia situación (Peláez \& García, 2014).

Entre los numerosos métodos y técnicas de gestión que están disponibles, no hay ninguno o ninguna que funcione bien en todas las situaciones. Si cada una de las teorías, conceptos o técnicas, fuera universalmente aplicable, no habría necesidad de directivos. Todo podría ser programado en un ordenador y se tomarían las decisiones y se solucionarían los problemas, automáticamente. Esto es lo que da lugar al llamado management situacional 
o de contingencia. El aspecto de contingencia de la gestión es lo que caracteriza el trabajo de los directivos, que han de tener capacidad para analizar una situación particular de modo que se identifiquen sus características dominantes y pueda ser diferenciada de otras situaciones, para luego escoger la solución más apropiada (Restrepo \& Arias, 2015).

\section{Las Funciones de la Gestión.}

Los directivos llevan a cabo las cinco funciones características del proceso de gestión:

i. Planificar, organizar, dotar de personal, dirigir y controlar,

ii. Partiendo de una secuencia lógica, aunque en la práctica, cualquiera de estas funciones puede ser considerada con independencia de las demás y en la secuencia que dicten las circunstancias (Perea, 2016).

1. Planificar: establecer objetivos globales que aúne las acciones de todos los empleados. Además de establecer objetivos se han de diseñar programas y calendarios que contribuyan a la consecución de los mismos. Los directivos del primer nivel suelen establecer metas y planes a corto plazo, por ejemplo, cuántas unidades debe producir el grupo en el día. Los directivos de nivel medio manejan unos objetivos o metas que, generalmente, tienen una proyección de un mes a un año. Por último, los directivos del nivel superior fijan objetivos y desarrollan planes más amplios para toda la organización, con un horizonte entre uno y cinco años.

2. Organizar: pretende resolver la cuestión de quién debe hacer qué, es responsabilidad de los directivos señalar las tareas y deberes que tienen que realizarse para que la organización alcance sus objetivos. También han de establecer las relaciones que deben existir entre los distintos puestos de trabajo, la dependencia o jerarquía que existirá entre ellos.

3. Dotar de personal: los puestos de trabajo que cuelgan de un organigrama carecen de significado hasta que son ocupados por personas que se suponen cualificadas para desarrollar las tareas asignadas a dichos puestos. Cuando los directivos realizan las gestiones necesarias para cubrir los puestos, están llevando a cabo la función de dotación de personal.

4. Dirigir: una vez que los planes están establecidos, se haya creado la estructura orgánica y cubierto los puestos de trabajo, la organización ya está preparada para ponerse en marcha, y para hacerlo necesita de la dirección. Los directivos dirigen (ordenan o instruyen) a los empleados en la realización de sus tareas; esto exige que además de ser unos expertos comunicadores, deben saber proporcionar la adecuada motivación y liderazgo.

5. Controlar: en el momento en que la organización se ha puesto en movimiento, hay que esperar que todos sus miembros realicen bien su trabajo, que los planes se cumplan y que se alcancen los objetivos fijados. Pero muchas veces esto no sucede, y hay que comprobar con cierta regularidad si las cosas se están desarrollando o no según las previsiones, y en esto consiste la función de control 
del directivo. Además, debe saber tomar las medidas correctoras necesarias en cada momento.

\section{Modelo de gestión.}

Se establece como modelo de gestión a la integración de diferentes actividades relacionadas con el talento humano, el valor añadido a estos es la asociación que se producen en los elementos que los integran para lograr una mejor comprensión de los mismos (Peláez, 2014). Existen tipo de modelos según criterios de clasificación: de alto rendimiento (basado en la implicación y orientación hacia el personal); orientados al control (basado en el control disciplinar y de gestión impuesto por la dirección) (Perea, 2016).

La existencia de diversos modelos de gestión hacia el talento humano hace suponer la importancia del personal en una organización. Muchos investigadores especialistas en el tema como Carachos, Barling e Iverson (2005), Zohar (2002) plantean modelos de gestión modernos basados al control, compromiso, implicación, rendimiento, seguridad en el trabajo y orientados al cliente (Majad, 2016).

Para mejorar las alternativas en modelos de gestión de talento humano desde una perspectiva estratégica y explicar las relaciones entre las dimensiones y sus consecuencias para la organización, Peláez y García (2014) considera que se debe elegir un modelo que permita avanzar en la comprensión de dos enfoques: que se alcance los objetivos organizacionales y se desarrolle el comportamiento social del empleado como estrategia que contribuye al desempeño positivo de la empresa.

Gracias al resultado del estudio de factores socioeconómicos condicionantes, se ha llegado a la concepción de diferentes modelos para la aplicación práctica de sistemas de gestión y cuya adopción dependerá del análisis de la situación específica de la empresa y del entorno (Aduna y García, 2014).

Los tipos de modelos de acuerdo al criterio de clasificación son por alto rendimiento que se basan en la implicación y la orientación hacia el personal de la organización y orientadas al control que se basan en el control disciplinar y de gestión impuesto por la dirección de la organización; por composición que se basan en las prácticas de recursos humanos empleadas; por objetivos que se intentan alcanzar con la aplicación de un determinado modelo (Rodríguez y Rodríguez, 2011).

\section{Modelo de gestión de calidad.}

La tendencia actual de la sociedad occidental tanto en el sector privado como en el público es la adopción de modelos de gestión que sirvan de referente y guía en los procesos permanentes de mejora de los productos y servicios que ofrecen. Un modelo es una descripción simplificada de una realidad que se trata de comprender, analizar y, en su caso, modificar (Rodríguez \& Rodríguez, 2011). 
Un modelo de referencia para la organización y gestión de una empresa permite establecer un enfoque y un marco de referencia objetivo, riguroso y estructurado para el diagnóstico de la organización, así como determinar las líneas de mejora continua hacia las cuales deben orientarse los esfuerzos de la organización. Es, por tanto, un referente estratégico que identifica las áreas sobre las que hay que actuar y evaluar para alcanzar la excelencia dentro de una organización (Rodríguez D. , 2012).

Un modelo de gestión de calidad es un referente permanente y un instrumento eficaz en el proceso de toda organización de mejorar los productos o servicios que ofrece. El modelo favorece la comprensión de las dimensiones más relevantes de una organización, así como establece criterios de comparación con otras organizaciones y el intercambio de experiencias. La utilización de un modelo de referencia se basa en que:

- Evita tener que crear indicadores, ya que están definidos en el modelo.

- Permite disponer de un marco conceptual completo.

- Proporciona unos objetivos y estándares iguales para todos, en muchos casos ampliamente contrastados.

- Determina una organización coherente de las actividades de mejora.

- Posibilita medir con los mismos criterios a lo largo del tiempo, por lo que es fácil detectar si se está avanzado en la dirección adecuada (Vieira, 2014).

Con el trascurso del tiempo el mercado consumidor de bienes y servicios se ha transformado en un mercado muy selectivo que, por ende, ha llevado a la oferta a proveer productos de mejor calidad y más competitivos. El fin que busca el cliente hoy en día es la entrega de calidad. Es necesario establecer una estructura de actividades en la organización con el propósito de conseguir este objetivo, todo este proceso se lo conoce como Gestión de la Calidad. "La calidad supone que el producto o servicio deberá cumplir con las funciones y especificaciones para las que ha sido diseñado y que deberán ajustarse a las expresadas por los consumidores o clientes del mismo. La competitividad exigirá, además, que todo aquello se logre con rapidez y al mínimo coste" (Cuatrecasas, 2010, pág. 12).

Es necesario hoy en día que las empresas que presenten problemas al momento de la entrega de servicio busquen la manera de corregir esta debilidad, ya que lo que busca el cliente actualmente es la calidad no solo del producto sino también la calidad de servicio entregada al momento de adquirir un producto. Por ello es que se ha vuelto más común el deseo de las empresas por mejorar sus procesos mediante la implementación de modelos de gestión de calidad (Borja \& Jijón, 2014).

La gestión de la calidad puede responder a normas comúnmente aceptadas. La normalización es importante para la sociedad "ya que facilita la adaptación de los productos, procesos y servicios a los fines a los que se destinan, protegiendo la salud y el medio ambiente, previniendo los obstáculos al comercio y facilitando la cooperación al comercio" (Lussier, 2012, pág. 44). 
Entenderemos modelo como una herramienta orientada a conseguir la mayor eficacia y eficiencia en la gestión, una descripción simplificada de la realidad que nos permite describirla, analizarla y modificarla.

Más complejo es definir que entenderemos por calidad, dado que sus definiciones son dinámicas y variadas. Si bien, y de acuerdo a los objetivos de este artículo, nuestro interés en la calidad apunta más bien al concepto en los servicios, veamos primero algunas definiciones más genéricas.

Calidad, en el marco del cumplimiento de estándares, se define como el nivel de acuerdo con las especificaciones establecidas en el diseño del producto o servicio, o calidad técnica. Esta definición esta más enmarcada en el ámbito de la producción manufacturera, y las primeras referencias al concepto.

Calidad como el conjunto de características de un producto que satisfacen las necesidades de los clientes. La calidad como la característica de un producto sin deficiencias. Calidad entendida como excelencia; la calidad implica obtener un estándar más alto o mejor resultados que otros que ofrecen el mismo producto o servicio.

En la práctica, en un primer momento las definiciones de calidad, no considerar no necesariamente la visión del cliente respecto al producto o servicio. Lo anterior tenía la limitante, de que, si bien la organización podría cumplir cabalmente con los estándares planeados, los clientes podrían nos sentirse satisfechos, pues los estándares no consideraban su opinión.

Es así como se derivó finalmente en definiciones, que entienden la calidad en el contexto de las percepciones de los receptores del producto o servicio. Calidad se define también como el valor que entrega un consumidor a un producto o servicio determinado (Borja \& Jijón, 2014).

\section{Componentes del modelo de gestión de calidad.}

\section{Calidad del servicio.}

La calidad del servicio ha pasado a ser un tema estratégico, existen varios autores que han propuesto escalas y dimensiones para la calidad percibida que es el resultado de la comparación entre lo que el cliente considera que el servicio ofrece (expectativa) y su percepción del desempeño del servicio entregado, sin embargo, esta propuesta también ha sido debatida por varios autores, descartando las expectativas de la medición (Vallejo \& Portalanza, 2017).

Rojas (2015) la han definido como la evaluación de la totalidad de aspectos y características de un producto o servicio que se relacionan con su habilidad de satisfacer las necesidades del cliente. Esto va más allá de que el producto tenga defectos o no, sino que más bien, la calidad se presenta a raíz las necesidades del cliente (lo esperado) y finaliza con la satisfacción de éste (valor percibido). Por lo mismo, tal como se vio, se 
puede concluir que tanto calidad y satisfacción se encuentran estrechamente relacionados, mas no corresponden a la misma dimensión.

Tabla 1. Dimensiones de la calidad del servicio y sus definiciones

\begin{tabular}{|l|l|}
\hline \multicolumn{1}{|c|}{ Criterios } & \multicolumn{1}{c|}{ Definiciones } \\
\hline Elementos tangibles & Apariencia de las instalaciones físicas. \\
\cline { 2 - 3 } Fiabilidad & $\begin{array}{l}\text { equipos, personal y materiales de comunicación. } \\
\text { Cuididad para ejecutar el servicio prometido de forma fiable y } \\
\text { cuidadosa }\end{array}$ \\
\hline $\begin{array}{l}\text { Capacidad de } \\
\text { respuesta }\end{array}$ & $\begin{array}{l}\text { Disposición de ayuda a los clientes y proveedores de un servicio } \\
\text { rápido. }\end{array}$ \\
\hline Profesionalidad & $\begin{array}{l}\text { Posesión de las destrezas requeridas y conocimiento de la ejecución } \\
\text { del servicio. }\end{array}$ \\
\hline Cortesía & $\begin{array}{l}\text { Atención, consideración, respeto y amabilidad de personal de } \\
\text { contacto. }\end{array}$ \\
\hline Credibilidad & Veracidad, creencia y honestidad en el servicio que se provee. \\
\hline Seguridad & Inexistencia de peligros, riesgos o dudas. \\
\hline Accesibilidad & Accesible y fácil de contactar. \\
\hline Comunicación & $\begin{array}{l}\text { Mantener a los clientes informados utilizando un lenguaje que puedan } \\
\text { entender, así como escucharles. }\end{array}$ \\
\hline $\begin{array}{l}\text { Comprensión del } \\
\text { cliente }\end{array}$ & Hacer el esfuerzo de conocer a los clientes y sus necesidades. \\
\hline
\end{tabular}

Fuente: Tomado de Rojas (2015)

A estas dimensiones, fueron añadidas 4 más (Comunicación boca a boca, Necesidades personales, Experiencias anteriores, Comunicación externa), las que conformarían el modelo de determinación de la calidad percibida.

Para estudiarla, algunos de los modelos de medición de la calidad de mayor difusión han sido el modelo de diferencias entre expectativas y percepciones, el modelo de las cinco dimensiones o criterios, el modelo integral de las brechas sobre la calidad del servicio, la escala de SERVQUAL ideada por los investigadores Valarie Zeithaml, A. Parasuraman y Leonard Berry, y la escala de Servpref elaborada por Cronin y Taylor (1994) (Rojas, 2015).

Figura 1. Evaluación del cliente sobre la calidad del servicio 


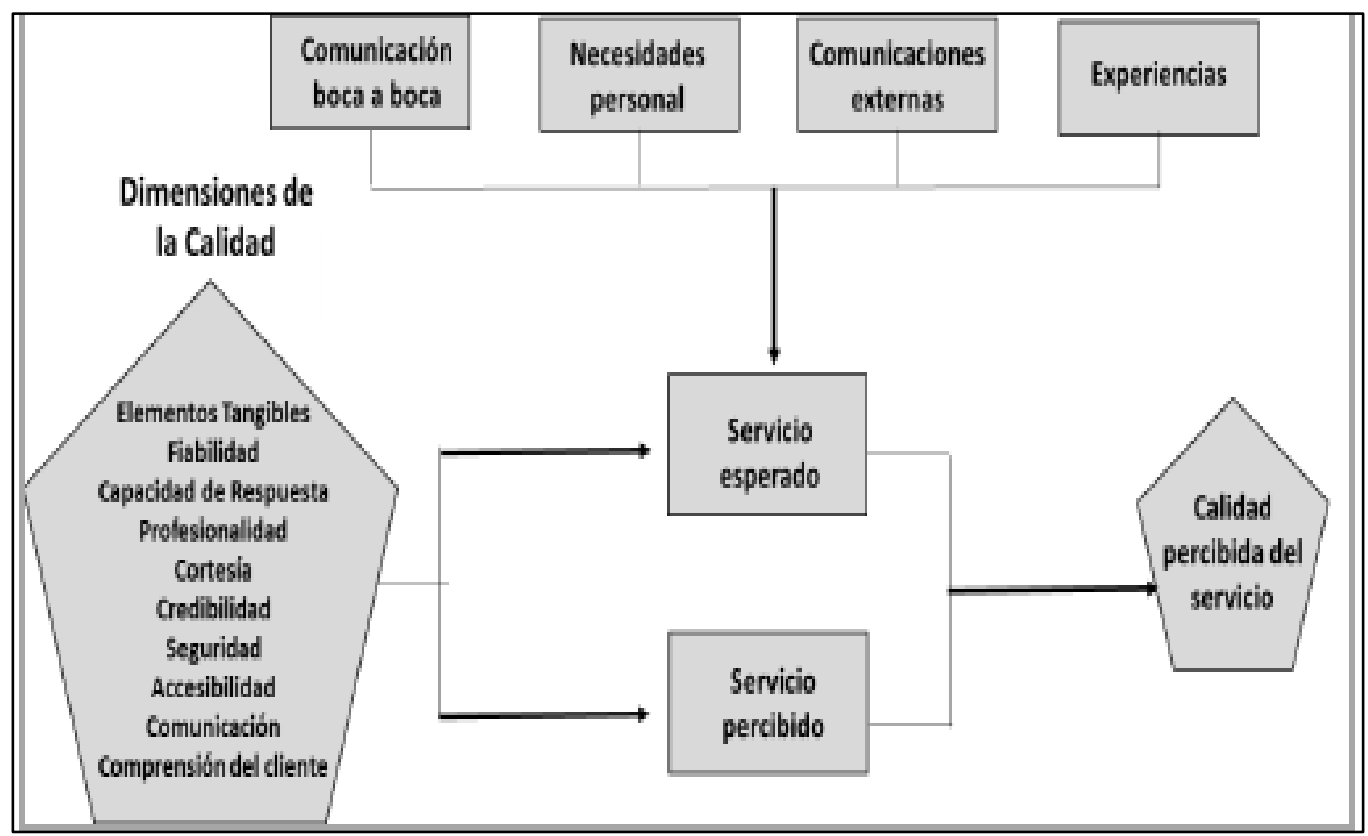

En la actualidad todos coinciden en reconocer la necesidad de mejorar la calidad de los productos y/o servicios para ser competitivos y obtener la permanencia, frecuentemente el tema en que no coinciden es la manera de lograrlo, algunos piensan que la mejora se dará con el solo hecho de exigir la calidad en el trabajo que desempeña cada uno de los miembros de la organización, es decir, piensan que es cuestión de imponer disciplina a los trabajadores. Sin embargo, el concepto de calidad es impreciso ya que las personas interpretan la calidad en forma diferente, muy pocos pueden definir la calidad en términos que sea posible medir y traducir en operaciones; esto se complica aún más tratándose de empresas de servicios (Huergo, 2016).

Es común que se diga que la calidad la define el cliente, lo cual es más complicado cuando el cliente es de diferentes segmentos de mercado. El cliente nos debe dar su juicio sobre lo que necesita, la forma en que lo necesita y el momento en que se lo debemos dar, al final es quien nos aprueba o rechaza el producto y/o servicio (Majad, 2016).

\section{Calidad de satisfacción.}

En general, la percepción de satisfacción que se ha dado cuenta en este estudio hace referencia a la percepción que tuvo el usuario de acuerdo a una experiencia, en la cual se vieron relacionadas sus necesidades más el resultado esperado y final. Se hacen referencia a la satisfacción como "la resultante sicológica de una experiencia de consumo, la que no debe confundirse con la evaluación de la experiencia, sino que debe entenderse como el balance psicológico y retrospectivo de la experiencia del consumo, donde la medida de satisfacción surge de la consistencia en las respuestas a una serie de cuestiones relativas al grado de bienestar que siente una persona por un fenómeno específico de vida, como es un servicio, un producto o una marca concreta" (Rojas, 2015). 


\section{Calidad de la Imagen.}

La imagen corresponde al "conjunto de rasgos y condiciones objetivas del ser social de la institución". Aquellos elementos reconocidos podrían establecerse como su identidad jurídica $y / o$ funcionamiento legal; estructura organizativa $y / u$ operativa; su realidad financiera; y la infraestructura en que se sustenta más sus recursos materiales. Desde este enfoque, las empresas han conseguido que el concepto de imagen se logre convertir en un activo importante para conseguir permanencia y fidelidad del mercado, preparándolas para enfrentar los constantes cambios que suceden en él (Rojas, 2015).

Figura 2. Relación causal entre factor imagen con factor satisfacción.

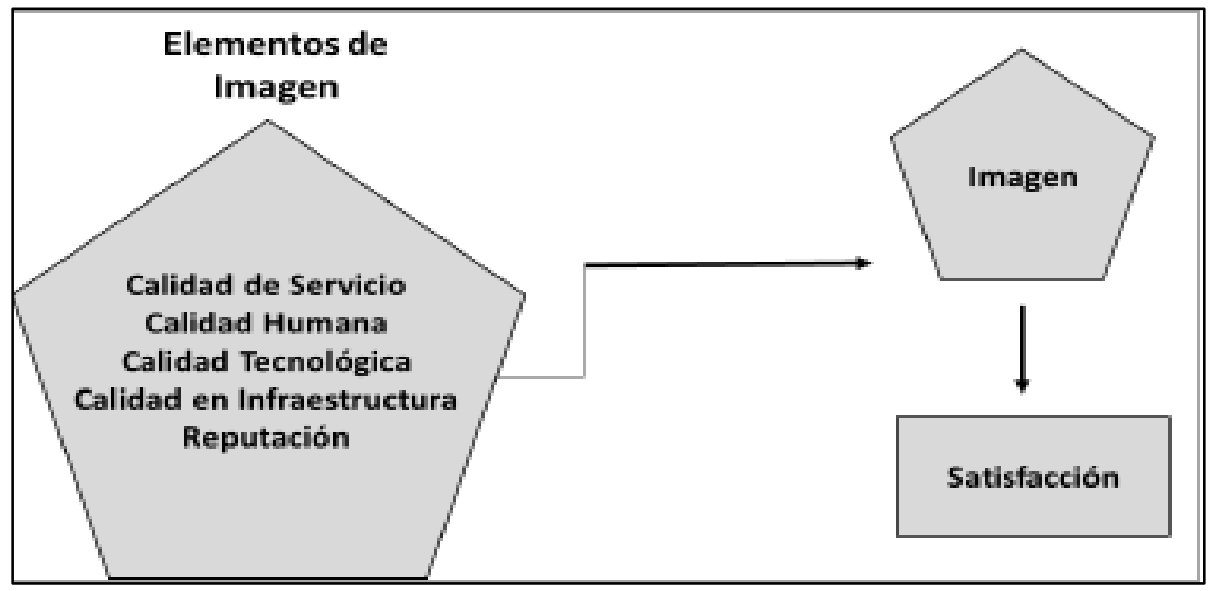

\section{Modelos de gestión de calidad de servicio con implicaciones estratégicas}

\section{El modelo de Brogowicz, Delene y Li}

Se propone un modelo de calidad de servicio en el que se hace especial hincapié en los aspectos de gestión, a la par que se sintetizan las aportaciones de las escuelas nórdica y norteamericana. Dicho modelo descansa sobre la idea de que los directivos deben prestar especial atención a la planificación, implementación y control tanto de la dimensión técnica como de la dimensión funcional de la calidad como vía para prevenir o, al menos, minimizar la diferencia entre la calidad esperada y la realmente recibida u ofrecida (Serrano \& López, 2007).

Analizando en mayor detalle el modelo, su núcleo central lo constituye precisamente el global de calidad de servicio que se determina a partir de las expectativas y percepciones de calidad de servicio de cada cliente real o potencial, siendo cada una de estas dos variables resultado de diferentes factores. En el caso de las expectativas, su proceso de formación se ve afectado por tres elementos básicos:

a) Las "influencias externas", concepto que engloba aspectos como la cultura, comunicaciones boca-oreja, competencia, necesidades personales y experiencias pasadas de los clientes,

b) Las "actividades tradicionales de marketing" que lleva a cabo la empresa, fundamentalmente, su plan de marketing-mix, y 
c) La "imagen corporativa" de la empresa, que se configura a partir de las interacciones de los dos anteriores.

En cuanto se refiere a las percepciones, son el resultado no sólo de la oferta global de servicio que hace la empresa, sino también de las expectativas previas del cliente y de los tres elementos anteriormente mencionados -influencias externas, actividades de marketing e imagen corporativa. Por lo tanto, la tarea de la dirección de la empresa se centra en determinar la misión y objetivos de la empresa para, a continuación, planificar, implementar y controlar las estrategias que permitan minimizar o eliminar la calidad de servicio. Para llevar a cabo esta tarea precisan de información sobre todos los aspectos previamente señalados, información que constituirá el punto de partida para diseñar la oferta global de servicio de la empresa (Cuatrecasas, 2010).

Llegados a este punto, hay dos dimensiones de calidad de servicio propuestas por la escuela nórdica, al entender que para intentar satisfacer las expectativas de los clientes los directivos han de planificar y controlar tanto la dimensión técnica del servicio que se traduce en especificaciones de rendimiento del sistema y en la que se incluyen tanto los conocimientos y habilidades de los recursos humanos como las máquinas, materiales, instalaciones y tecnología que son necesarios para realizar el servicio como la dimensión funcional del servicio que traducen en especificaciones de rendimiento de las operaciones y en la que se tienen en cuenta respecto a los recursos humanos la disposición hacia el servicio, la disponibilidad del personal o la calidad de las relaciones internas, y respecto a los recursos físicos su apariencia y accesibilidad-. Por otra parte, ambos tipos de especificaciones exigen tener en cuenta los recursos, humanos y físicos, disponibles (Lussier, 2012).

A partir de todas estas premisas, la dirección determina tanto la oferta técnica como la oferta funcional de servicio. Conjuntamente consideradas, éstas constituyen la oferta global de servicio que hace la empresa con el objetivo de minimizar riesgo de servicio global, entendido como resultado de la existencia de dos riesgos que influyen sobre el mismo: calidad técnica de servicio y el de calidad funcional (Peláez, 2014).

\section{Conclusión.}

- La calidad del servicio es muy importante en uno de los logros de los objetivos institucionales tanto de los clientes internos como externos..

- Buscar el mejoramiento continuo y llegar a la satisfacción plena de los clientes es uno de los objetivos de este artículo.

- Beneficiar a las instituciones estatales que trabajan de manera coordinada con la Corporación y que en muchas ocasiones reciben reclamos de clientes, tales como Gobernaciones. Municipios, CONELEC y Ministerio de Electricidad y Energía Renovable.

- Buscar coordinar y llegar a cumplir con los objetivos institucionales sería el fin de esta investigación. 


\section{Referencia Bibliográfica.}

Aduna, A., \& García, E. (2014). Modelos de gestión de Recursos Humanos. Universidad Autónoma del Estado de Hidalgo, 52-69.

Aguilar, J. C. (2014). Retos y rol estratégico en la gestión del talento Humano Caso en empresas de Cali, Colombia. MERCADOS y Negocios. Volumen 15, núm. 1, 420.

Borja, S., \& Jijón, J. (2014). Propuesta de un modelo de gestión en calidad de servicio, basado en la norma internacional ISO 9001: 2008, en empresas de comercialización de productos de consumo masivo, caso: almacenes la rebaja. Quito - Ecuador: Universidad Internacional del Ecuador.

Campuzano, M., Ziadet, E., \& Echeverría, H. (2016). Gestión del Talento Humano en las PYMES. Revista Publicando. Vol. 3, N 7, 438-448.

Cuatrecasas, L. (2010). Gestión integral de la calidad: Implantación, control y certificación. Profit Vértice Vol09 N 5, 10-25.

Huergo, J. (2016). Los procesos de gestión. Universidad pedagógica. Vol 12, No 14, 1-5.

Lussier, R. (2012). Liderazgo: teoría, aplicación y desarrollo de habilidades. UOC Psicología de las organizaciones, 42-61.

Majad, M. (2016). Gestión del talento humano en organizaciones educativas. Revista de Investigación $\mathrm{N}^{\mathrm{o}} 88$ Vol. 40, 148-165.

Peláez, J. (2014). La responsabilidad social empresarial y la gestión humana en Colombia: desafíos para fortalecer una relación estratégica. Ciencias estratégicas Vol. 31, No 23, 74-85.

Peláez, J., \& García, M. (2014). Responsabilidad social empresarial y gestión humana: una relación estratégica aplicada desde un modelo explicativo. Entramado, vol. 10, núm. 2, 90-111.

Perea, J. L. (2016). Gestión de recursos humanos: enfoque sistémico en una perspectiva global. Revista IIPSI. Facultad de Psicología UNMSM Vol 19, № 1, 109-122.

Restrepo, F., \& Arias, F. (2015). Las prácticas de gestión del talento humano en empresas agropecuarias del sector bananero en Colombia. Journal of Agriculture and Animal Sciences Vol. 4, No 2., 20-32.

Rodríguez, A., \& Rodríguez, M. (2011). MOdelos de gestión de recursos humanos. Psicología de las organizaciones, Universidad de Oviedo, 19-35.

Rodríguez, D. (2012). Prácticas de gestión humana en pequeñas empresas. Apuntes del CENES, Vol. 31, No 54, 193-226.

Rojas, J. (2015). Un modelo de satisfacción de usuarios como herramienta de apoyo a la gestión de una municipalidad: análisis de los servicios entregados en edificio consistorial y departamento de desarrollo social de la municipalidad de Lo Prado. Santiago de Chile: Universidad de Chile.

Serrano, A., \& López, M. C. (2007). Modelos de gestión de la calidad de servicio: revisión y propuesta de integración con la estrategia empresarial. Universidad de Cantabria, 1-9.

Uranga, W. (2015). Gestionar la comunicación, en las prácticas sociales, organizaciones y comunidades. Labor pedagógica. Vol 2, No 3, 19-33. 
Vallejo, V., \& Portalanza, A. (2017). Importancia de la Gestión del Talento Humano como Estrategia para la Atracción y Retención de Docentes en las Organizaciones Educativas de Ecuador. PODIUM Edición Especial, abril , 145-168.

Vieira, C. (2014). Gestión de recursos humanos: indicadores y herramientas. Observatorio Laboral Revista Venezolana. Vol. 7, No 14, 23-33.

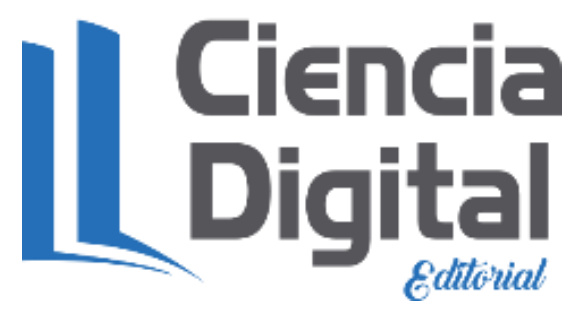


Para citar el artículo indexado.

Campos L. \& Lazo C. (2019). La calidad del servicio en la mejora de la gestión en la atención al usuario de CNEL EP del cantón Milagro-Ecuador en el año 2018. Revista electrónica Visionario Digital 3(2), 99-113. Recuperado desde: http://www.cienciadigital.org/revistascienciadigital/index.php/VisionarioDigital/article/view/3 $\underline{95 / 887}$

\section{Liencia}

El artículo que se publica es de exclusiva responsabilidad de los autores y no necesariamente reflejan el pensamiento de la Revista Ciencia Digital.

El articulo queda en propiedad de la revista y, por tanto, su publicación parcial y/o total en otro medio tiene que ser autorizado por el director de la Revista Ciencia Digital.
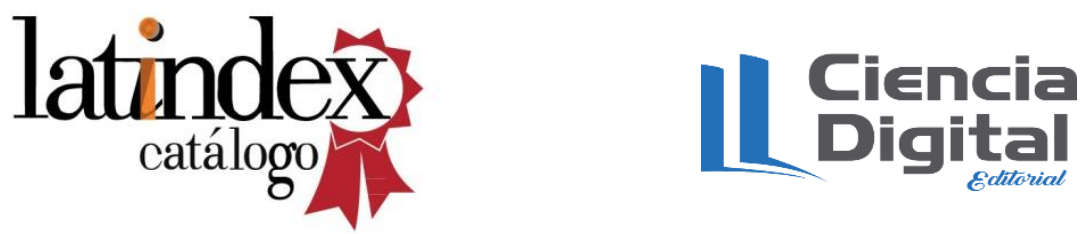